\title{
The growing burden of multidrug- resistant infections among returned Australian travellers
}

\author{
Kyra Y L Chua \\ Microbiology Registrar \\ M Lindsay Grayson \\ FRACP, MD, \\ Director of Infectious \\ Diseases \\ Adele N Burgess \\ FRACS, \\ Head of Colorectal Surgery \\ Jean Y H Lee \\ MB BS, \\ Infectious Diseases \\ Registrar \\ Benjamin P Howden \\ FRACP, FRCPA, \\ Infectious Diseases \\ Physician \\ Austin Health, \\ Melbourne, VIC \\ benjamin.howden@ \\ austin.org.au
}

doi: 10.5694/mjal3.10592

\section{Clinical record}

A previously well 66-year-old man was repatriated from Athens, Greece, to the Austin Hospital for ongoing management after a protracted hospital admission for an ischiorectal abscess secondary to perforated diverticulitis. This was complicated by faeculent peritonitis, multiple intra-abdominal abscesses and necrotising fasciitis of the abdominal wall. These complex problems required multiple laparotomies to drain and debride the abscesses, management of an open abdomen with vacuum-assisted closure dressings, and the formation of a loop sigmoidostomy. He also developed a grade IV sacral pressure ulcer with underlying sacral osteomyelitis. Organisms isolated from the intra-abdominal collections included carbapenemresistant Pseudomonas aeruginosa and a carbapenemaseproducing Klebsiella pneumoniae ( $b / a_{K P C}$ ). Due to the complexity of the patient's illness, he had spent 93 days in hospital in Greece, predominantly in intensive care, with three interhospital transfers within Greece before repatriation to Australia. Antibiotics administered in Greece included tigecycline, colistin, fosfomycin, vancomycin, clindamycin and anidulafungin.

As the patient had multiple resistant organisms, detailed infection control plans were made before his arrival at the Austin Hospital. This included placement in a single room with a dedicated ensuite bathroom, daily bleach cleaning of the room,' no use of shared equipment, enforcement of strict contact precautions including gowns and gloves, and hand hygiene. Patient movement was severely restricted and only two visitors were allowed at any one time.

Unfortunately, the patient developed a new intra-abdominal collection, bowel obstruction and abdominal sepsis. This required surgical intervention, including extensive division of adhesions, resection of the sigmoid and part of the descending colon, retroperitoneal enteric fistula repair and retroperitoneal

ultidrug-resistant (MDR) gram-negative bacteria have emerged as a global health threat. The index case of a repatriated patient with complex MDR Klebsiella pneumoniae infection prompted a retrospective review of similar admissions to our institution between December 2011 and February 2013. Austin Health is an 800bed hospital over three campuses with 98125 admissions yearly. It is the statewide referral centre for liver transplantation and the spinal cord service.

The case definition for this review was: a patient who had travelled overseas in the 6 months before admission to Austin Health, had a length of stay at Austin Health of $\geqslant 2$ weeks, and had infection or colonisation with MDR gramnegative organisms. ${ }^{2}$ Although all patients who are transferred from another hospital to Austin Health are systematically screened for the presence of MDR organisms, the case abscess drainage. An end colostomy and loop ileostomy were formed. This procedure resulted in faecal continence and therefore control of the perianal source of multidrug-resistant organisms. Culture of the intra-abdominal abscess grew mixed enteric flora including Enterococcus faecium, Escherichia coli, Citrobacter spp, Candida glabrata and $K$. pneumoniae. The latter organism was resistant to multiple drugs, including meropenem, due to the production of $K$. pneumoniae carbapenemase- 2 (bla $a_{\mathrm{KPC}-2}$ ) (Patient 1, Box). The same organism was found in his faeces.

A bla $a_{\mathrm{KPC}-2}$-producing $K$. pneumoniae was also isolated from a sacral ulcer swab, but the susceptibility profile was slightly different. This isolate was also resistant to all aminoglycosides, including gentamicin and amikacin, and demonstrated an increased minimum inhibitory concentration to colistin (Box). The patient's antibiotic treatment included meropenem, tigecycline, colistin and caspofungin for 6 weeks, and his sacral ulcer was treated with a vacuum-assisted closure dressing. He stayed at the Austin Hospital for 101 days before being discharged home.

Six months later, the patient re-presented to the Austin Hospital with urosepsis. The causative organism, isolated in both urine and blood, was E. coli (Box). Strikingly, the organism was found to be a bla KPC-2$_{2}$-producing strain, suggesting interspecies transfer of this mobile genetic element between $K$. pneumoniae and $E$. coli. Unlike the $K$. pneumoniae, this isolate was susceptible to ciprofloxacin, and the patient was successfully treated with this antibiotic. During this second admission, the same infection control measures were enforced.

At follow-up 6 months later, the patient remained well. There was no documented inhospital transmission of $b_{l} a_{\mathrm{KPC}-2}$, suggesting the infection control measures employed were successful.

finding in this retrospective review was largely opportunistic. This case review was approved by the Austin Health Office for Research as an audit activity project.

Screening rectal swabs or faecal samples were plated onto chromID ESBL media (bioMérieux). Clinical specimens were processed routinely. Organism identification and susceptibility testing were performed using agar dilution or the VITEK 2 automated system (bioMérieux). Minimum inhibitory concentrations to tigecycline and colistin were determined using Etest (bioMérieux). Gramnegative isolates were classified as MDR, extensively drugresistant (XDR) or pandrug-resistant, according to international guidelines. ${ }^{2}$ Where testing against a particular agent was not done, the result was recorded as susceptible for that agent to avoid overestimating the burden of resistance. Genotypic detection of resistance was performed at 


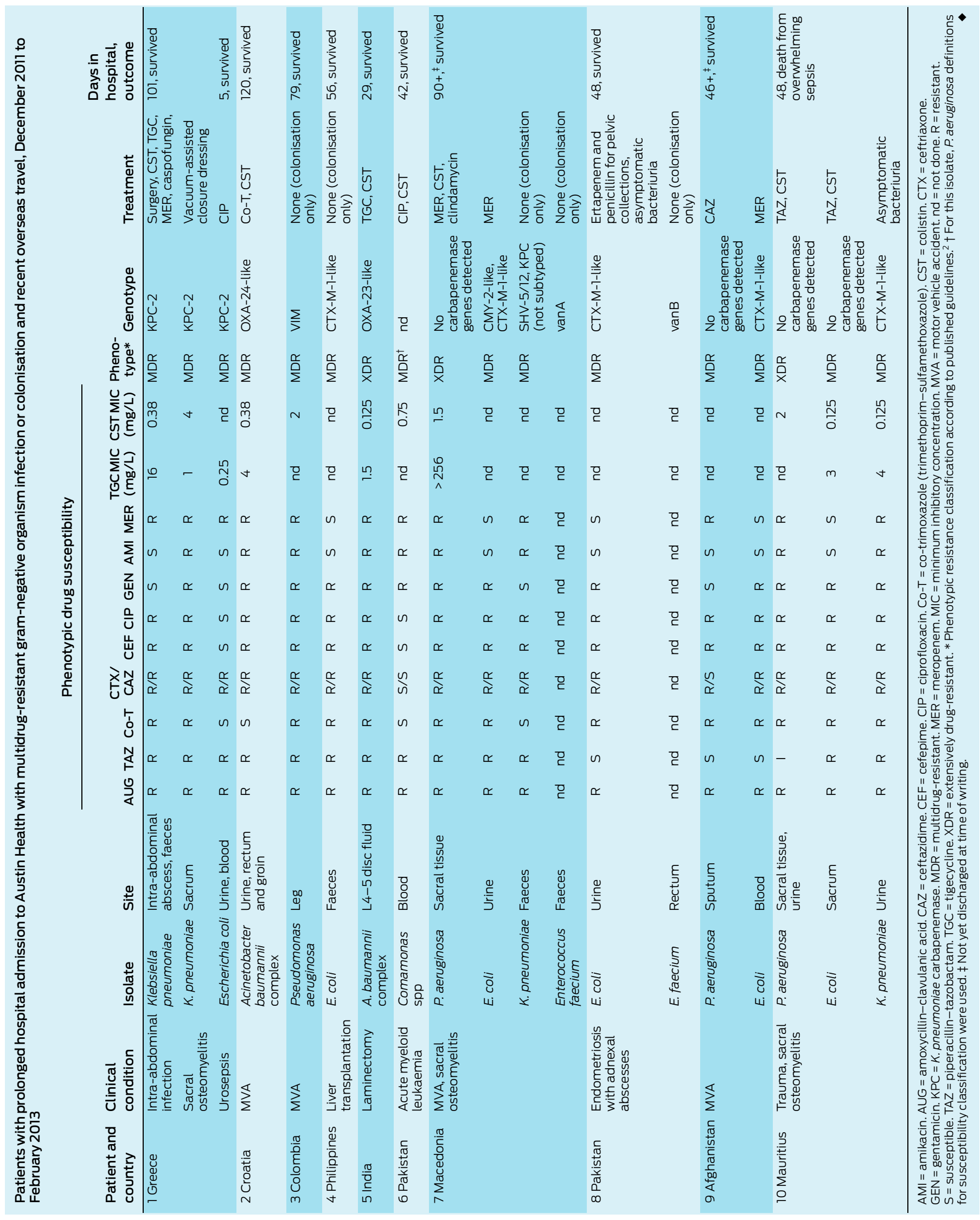


Pathology West laboratories using multiplex polymerase chain reactions (PCRs). ${ }^{3}$

We identified 10 patients during the study period (Box). For all patients except Patient 6, who developed bacteraemia after admission, MDR gram-negative organisms were detected on admission. Eight patients had either been repatriated directly to Australia or discharged from hospitals overseas. There was no predominant country of travel, with a wide geographical spread over multiple continents. The most common clinical problems, occurring in five patients, were accidents resulting in spinal cord injuries or multiple fractures. A broad range of antibiotics was used to treat the infections, with colistin being the most common. Colistin is an old drug that has been "rediscovered" with the advent of MDR gram-negative infections. ${ }^{4}$ It is associated with significant nephrotoxicity, which was seen in four of six patients. Nine patients survived; one died from overwhelming sepsis related to MDR Pseudomonas aeruginosa sacral osteomyelitis.

Each patient with a carbapenem-resistant organism was managed in a single room with strict contact precautions. When a patient was transported for procedures, all surfaces in contact with the patient were cleaned with 1:1000 ppm of chlorine-based disinfectant. For most patients, this occurred before the microbiological test results were available, as patients who had been recently hospitalised overseas were "red-flagged" by the bed manager and infection control team. There were no identified outbreaks resulting from any of these patients.

In these 10 patients, 19 MDR organisms were isolated. Gram-negative organisms $(n=17)$ were far more common than gram-positive organisms. Most of the gram-negative isolates were classified as MDR, but three isolates ( $P$. aeruginosa and Acinetobacter baumannii complex) were XDR (Box). Of concern, 12 isolates were meropenem-resistant. Most drug-resistant gram-negative organisms were judged clinically to be causing infection and requiring treatment, with only four deemed as colonisers. In addition to the burden of disease in each patient, the cost to the health care system was significant. The total duration of hospitalisation for each patient ranged between 29 and 120 days (mean, 66.4 days). Although difficult to quantify, additional costs incurred from enforcement of strict contact precautions included those related to personal protective equipment, cleaning and prolongation of length of stay due to limits placed on rehabilitation.

Infections with MDR gram-negative bacteria are now well described worldwide. ${ }^{4}$ The rapidity of their spread, coupled with a lack of new antimicrobial agents, is alarming. ${ }^{5}$ Of greatest concern is the rise of carbapenemaseproducing organisms, as carbapenems are the last line of commonly available gram-negative antibiotics. ${ }^{4}$ Infections associated with these organisms have high mortality (51\%$59 \%$ ) and have been associated with numerous health care-associated outbreaks. ${ }^{4-6}$

In Australia, we are fortunate that such organisms are not yet endemic, but many of these organisms and their resistance mechanisms have been reported locally. ${ }^{5,7}$ With increasing international travel, these resistant bacteria are no longer limited by geographical boundaries. In 2012, there were a record 8.2 million short-term resident departures from Australia. ${ }^{8}$ Among the top 10 destination countries were our neighbours including Indonesia, Thailand and

\section{Lessons from practice}

Hospitals should follow recommendations for preventing institutional spread of multidrug-resistant (MDR) gram-negative organisms. 11,12

Patients at high risk of colonisation or infection with MDR gram-negative bacteria should be identified; hospitalisation overseas is a key risk factor.

All high-risk patients should be isolated, pending results of screening cultures. Strict infection control measures should be implemented, including contact precautions, hand hygiene, patient placement in single rooms, intensive cleaning of the environment, restriction of patient movement and minimising visitor contact. Screening protocols for colonisation in high-risk patients should be followed. Appropriate patient specimens include rectal or perianal swabs or faeces.

China. Recent data from China show hospital rates of carbapenem resistance exceeding $50 \%$ in Acinetobacter and $25 \%-30 \%$ in P. aeruginosa. ${ }^{9}$

A recent Australian study examining the risk of resistant $E$. coli after international travel showed that colonisation increased from $7.8 \%$ to $49 \% .{ }^{10}$ The locations most frequently associated with acquisition of resistant $E$. coli were the Indian subcontinent, China, the Middle East and Africa. In contrast, most cases in our study were clinical infections rather than colonisation, indicating that the threat of MDR gram-negative bacteria is no longer just a theoretical problem. To document the problem and direct resources to this growing threat at a national level, strong consideration should be given to making these infections notifiable. ${ }^{5,11}$

Although this case review was limited by the lack of denominator data, there appears to be a high risk of infection or colonisation with MDR organisms in patients who are repatriated from countries with high rates of resistance. It is imperative that hospitals that accept such patients have management protocols in place to prevent spread of MDR organisms locally. ${ }^{11}$

Acknowledgements: We thank the patient for allowing us to publish details of his case, and staff at Pathology West for performing ESBL, AmpC and carbapenemase multiplex PCRs.

Competing interests: Benjamin Howden has been an advisory board member for Pfizer.

1 Grabsch EA, Mahony AA, Cameron DR, et al. Significant reduction in vancomycin-resistant enterococcus colonization and bacteraemia after introduction of a bleach-based cleaning-disinfection programme. J Hosp Infect 2012; 82: 234-242.

2 Magiorakos AP, Srinivasan A, Carey RB, et al. Multidrug-resistant, extensively drug-resistant and pandrugresistant bacteria: an international expert proposal for interim standard definitions for acquired resistance. Clin Microbiol Infect 2012; 18: 268-281.

3 Ellem J, Partridge SR, Iredell JR. Efficient direct extended-spectrum -lactamase detection by multiplex realtime PCR: accurate assignment of phenotype by use of a limited set of genetic markers. J Clin Microbiol 2011; 49: 3074-3077.

4 Tzouvelekis LS, Markogiannakis A, Psichogiou M, et al. Carbapenemases in Klebsiella pneumoniae and other Enterobacteriaceae: an evolving crisis of global dimensions. Clin Microbiol Rev 2012; 25: 682-707.

5 Looke DF, Gottlieb T, Jones CA, Paterson DL. Gram-negative resistance: can we combat the coming of a new "Red Plague"? Med J Aust 2013; 198: 243-244.

6 Souli M, Galani I, Antoniadou A, et al. An outbreak of infection due to beta-lactamase Klebsiella pneumoniae carbapenemase 2-producing K. pneumoniae in a Greek University Hospital: molecular characterization, epidemiology, and outcomes. Clin Infect Dis 2010; 50: 364-373.

7 Turnidge JD, Gottlieb T, Mitchell D, et al; Australian Group for Antimicrobial Resistance. Gram-negative survey 2011 antimicrobial susceptibility report. http://www.agargroup.org/files/AGAR\%20GNB11\%20Report\% 20FINAL.pdf (accessed Mar 2013).

8 Australian Bureau of Statistics. Overseas arrivals and departures, Australia, Dec 2012. Canberra: ABS, 2013. (ABS Cat. No. 3401.0.) http://www.abs.gov.au/AUSSTATS/abs@.nsf/Previousproducts/

3401.0Feature\%20ArticleIDec\%202012?opendocument\&tabname=Summary\&prodno=3401.0\&issue= Dec\%202012 (accessed Mar 2013).

9 Yezli S, Li H. Antibiotic resistance amongst healthcare-associated pathogens in China. Int J Antimicrob Agents 2012; 40: 389-397.

10 Kennedy K, Collignon P. Colonisation with Escherichia coli resistant to "critically important" antibiotics: a high risk for international travellers. Eur J Clin Microbiol Infect Dis 2010; 29: 1501-1506.

11 Australian Commission on Safety and Quality in Health Care. Recommendations for the control of multidrug resistant gram-negatives: carbapenem resistant Enterobacteriaceae. Sydney: ACSQHC, 2013. http:// www.safetyandquality.gov.au/publications/mrgn-guidelines-enterobacteriacea (accessed Dec 2013).

12 Kollef MH, Golan Y, Micek ST, et al. Appraising contemporary strategies to combat multidrug resistant gramnegative bacterial infections - proceedings and data from the Gram-Negative Resistance Summit. Clin Infect Dis 2011; 53 Suppl 2: S33-S55; quiz S6-S8. 